\title{
Enhanced immunotherapeutic effect of modified HPV16 E7- pulsed dendritic cell vaccine by an adeno-shRNA-SOCS1 virus
}

\author{
YONGQIANG ZHU ${ }^{1,2^{*}}$, YI ZHENG ${ }^{1,2^{*}}$, LIN MEI $^{1,2}$, MENGQIONG LIU ${ }^{3}$, SHANSHAN LI $^{4}$, HUAWEI XIAO ${ }^{1,2}$, \\ HUIJUN ZHU ${ }^{1,2}$, SHU WU ${ }^{1,2}$, HONGBO CHEN $^{1,2}$ and LAIQIANG HUANG ${ }^{1,2}$ \\ ${ }^{1}$ School of Life Sciences, Tsinghua University, Beijing 100084; ${ }^{2}$ The Shenzhen Key Laboratary of Gene and \\ Antibody Therapy, Center for Biotech and BioMedicine, State Key Laboratary of Health Sciences and \\ Technology, Division of Life and Health Sciences, Graduate School at Shenzhen, Tsinghua University, \\ Shenzhen, Guangdong 518055; ${ }^{3}$ Clinical Laboratory of Hainan Provincial People's Hospital, 570311, P.R. China; \\ ${ }^{4}$ Department of Plant Pathology and Microbiology, Iowa State University, Ames, IA 50011, USA
}

Received March 31, 2013; Accepted May 23, 2013

DOI: 10.3892/ijo.2013.2027

\begin{abstract}
Cervical cancer is the second most common cause of cancer-related deaths among women worldwide. However, no efficient therapy exists against cervical cancer and current treatments have several disadvantages. One possible novel approach is to develop immune-based strategies using tumor antigen-loaded dendritic cells (DCs) for the induction of cellular antitumor immunity. In this study, we created a modified HPV16 E7, HPV16mE7, to reduce its transformation activity and to enhance its antigenicity. The siRNA delivery technique was used to silence the suppressor of cytokine signaling 1 (SOCS1) gene in DCs. BM-derived DCs infected by ad-shRNASOCS1 were pulsed with the HPV16mE7 protein and then were transfused into mouse models bearing TC-1 tumor cells expressing HPV16 E6/E7. IFN- $\gamma$, cytokine (TNF- $\alpha$, IL-12, IL-6) expression, anti-E7 antibody and cytotoxic T lymphocyte (CTL) levels were measured. The survival rate, survival days and the tumor volume of the mouse models from the different treatment groups were monitored. The data showed that the mE7-pulsed DC vaccine enhanced by adenovirus-mediated SOCS1 silencing exhibited better immunotherapeutic effect on the allografted tumor mouse models. The method by silencing SOCS1 in HPV16mE7 protein-pulsed DCs may provide a new strategy for the development of safe and effective immunotherapy for cervical cancer.
\end{abstract}

Correspondence to: Professor Laiqiang Huang or Dr Yi Zheng, School of Life Sciences, Tsinghua University, Beijing 100084, P.R. China; and Graduate School at Shenzhen, Tsinghua University, L-411/403 Tsinghua Campus, University Town, Shenzhen, Guangdong 518055, P.R. China

E-mail: huanglq@tsinghua.edu.cn; huanglq@sz.tsinghua.edu.cn E-mail: zhengy@sz.tsinghua.edu.cn

*Contributed equally

Key words: human papillomavirus, suppressors of cytokine signaling 1, dendritic cell, vaccine, cervical cancer

\section{Introduction}

Cervical cancer is a major problem in women's health worldwide and is the second leading cause of cancer deaths in women (1). Clinical, molecular and epidemiological investigations identify the high-risk human papillomavirus (HPV) as the major cause of cervical cancer, with the high-risk types HPV16 and 18 accounting for nearly $70 \%$ of cervical cancer cases (2-5). The E6 and E7 oncoproteins encoded by these two viruses, are crucial for the productive viral life cycle in the transformation and maintenance of the malignant phenotype (6). Therefore, various forms of HPV vaccines targeting the two proteins are designed for cervical cancer immunotherapy, including plasmid DNA, viral or bacterial vectors, chimeric virus-like particles (VLP), synthetic peptides and recombinant proteins (3). Cervical cancer therapeutic vaccines face many challenges, including immunocompromised state of cancer patients, difficulty in immune system stimulation, immune escape and tolerance mechanisms used by tumors and virally infected cells, as well as safety concerns $(2,7)$. Thus, an emerging need for the treatment of cervical cancer and other HPV infection-associated diseases is required.

Dendritic cells (DCs) are the most potent professional antigen-presenting cells (APC) with key regulatory roles in immune response. It maintains tolerance to self-antigens and activates innate and adaptive immunity against viral infection by producing various proinflammatory cytokines, which is involved in processing and presenting antigens to $\mathrm{T}$ cells $(6,8)$. Recent studies on dendritic cell (DC)-based tumor vaccine demonstrated that enhancing DC maturation/co-stimulation and antigen presentation can greatly enhance human CTL and Th immune response in cancer therapy, suggesting a promising way to treat HPV and other cancers (8-13). Hence, activation of the full immunostimulatory potential of DCs to achieve an effective immune response provides a promising way to prevent or control many viral infections.

Recently, a major immunosuppressive, the intracellular signal regulator suppressors of cytokine signaling 1 (SOCS1) was found to play an important role in blocking the constitutive 
activation of the immune response (14). SOCS1 functions as an inducible negative feedback inhibitor of the JAk/STAT signal pathway (14) and negatively regulates various cytokines, such as interferon (IFN)- $\gamma$, the interleukin (IL)-2, IL-6, IL-7, IL-12, or IL-15 in T cells, DCs and other cells (15).

Several studies demonstrate that ex vivo SOCS1 silencing in antigen-presenting DCs can strongly enhance antigenspecific antitumor and antivirus immunity $(8,13,16,17)$. Hanada et al (18) and Guenterberg et al (19) showed that the antitumor activity of exogenous IFN- $\alpha$ was enhanced by $\mathrm{CD}^{+}$ and $\mathrm{CD}^{+} \mathrm{T}$ cells in $\mathrm{SOCS1}^{-/-}$transgenic mice and $\mathrm{T}$ and $\mathrm{B}$ cells were restored via exogenous SOCS1 expression. SOCS1 ${ }^{-1-}$ DCs were also hyper-responsive to LPS and IFN- $\gamma$, which triggers an allogeneic T-cell expansion. In addition, studies in macrophages demonstrated that silencing SOCS1 can improve Mycobacterium tuberculosis control and suppress tumor development by enhancing antitumor inflammation (20). All these findings indicate that SOCS1 possessed an inhibitory role in controlling antigen presentation by APCs and magnitude of the adaptive immunity. The regulation of antigen presentation and development of more effective tumor vaccines can therefore be achieved by silencing the crucial 'natural inhibitor' in antigen-presenting DCs.

DC vaccines combined with SOCS1 silencing has been previously reported for antitumor and antivirus purpose $(13,16)$. In this study, we sought to find a safer and more effective approach to treat cervical cancer and other HPV infectionassociated malignancies by constructing an HPV16 E7 mutant, the HPV16mE7, to reduce transformation activity and enhance antigenicity (21-23). The immunotherapeutic effect of mE7-pulsed DC vaccine with adenovirus-mediated SOCS1 silencing on the allografted tumor mouse models which express HPV16E6E7 oncoproteins, was investigated.

\section{Materials and methods}

Peptide synthesis of E7.49-57 and HBV surface antigen (HBsAg) R187. The synthetic peptides E7.49-57 (RAHYNIVTF) corresponding to the HPV16-E7 H2-Db restricted epitope (24) and HBV surface antigen (HBsAg) R187 (aa183-191) (FLLTRILTI) (25) were synthesized and purified to $>95 \%$ purity by Sangon Inc. (Shanghai, China) via high-performance liquid chromatography (HPLC).

Construction of modified HPV16 E7 gene, protein expression and purification. The HPV16 E7 mutant gene (HPV16mE7) was designed to reduce its transformation activity and enhance its antigenicity $(21-23,26)$. The three arbitrarily designated regions, a, b and c, of the native 98-amino acid HPV16 E7 protein were mutated as follows: in region a, amino acids 21 , 24 and 26 were mutated from DLYCYEQ to GLYGYGQ. In region $\mathrm{c}$, two zinc finger-binding motifs were disrupted by mutating $\mathrm{C} 61 \rightarrow \mathrm{G} 61$ and $\mathrm{C} 94 \rightarrow \mathrm{G} 94$ using a site-directed mutagenesis kit according to the manufacturer's instructions (Promega Co., Madison, WI, USA). The HPV16mE7 was cloned into the plasmid pET-28a vector (Invitrogen, Carlsbad, CA, USA) and then transformed into the Escherichia coli strain BL21 $\gamma$ (DE3) (Novagen). The recombinant protein containing His-Tag was expressed upon induction with $0.5 \mathrm{mM}$ isopropyl- $\beta$-D-thiogalactopyranoside (IPTG, Sigma,
St. Louis, MO, USA) and purified on Ni-NTA resin (Qiagen, Hilden, Germany). Isopropanol washes were used to remove lipopolysaccharide (LPS) contamination. HPV16mE7 protein was confirmed by western blot analysis probed either with mouse monoclonal anti-HPV16-E7 antibody (Zymed, San Francisco, CA, USA) or mouse anti-His polyclonal antibody. Immune complexes were detected with goat HRP-conjugated anti-mouse immunoglobulins (Chemicon, Temecula, CA, USA) and the result was revealed via electrochemical luminescence (ECL, Pierce Biotechnology, Inc., Rockford, IL, USA). HPV16 E7 concentration was measured by Bradford assay.

Adenovirus vector production. The shRNA-SOCS1 and its mutant form shRNA-mSOCS1 were constructed according to http://www.bioon.com.cn. Primers used are as follows: shRNASOCS1 (F): 5'-GATCC CTA CCT GAG TTC CTT CCC CT TCAAGAG AG GGG AAG GAA CTC AGG TAGTTTTTT G-3' (BamHI and EcoRI); shRNA-SOCS1 (R): 5'- $\underline{\text { AATTC }}$ AAAAAACTA CCT GAG TTC CTT CCC CT CTCTTGA AG GGG AAG GAA CTC AGG TAG G-3'; shRNA-mSOCS1 (F): 5'-GATCC ACT ATC TAA GTT ACT ACC CCT TCAAGAG AGG GGT AGT AAC TTA GAT AGT TTTTTTG -3'; shRNA-mSOCS1 (R): 5'-AATTC AAAAAAACT ATC TAA GTT ACT ACC CCT CTCTTGA AGG GGT AGT AAC TTA GAT AGT G-3'. The shRNA-SOCS1 and shRNA-mSOCS1 were cloned into the plasmid vector RNAi-SOCS1-pShuttle (BD Clontech) and then inserted into the replication-deficient pAdeno-X vector (BD Clontech). The recombinant adenovirus plasmids were generated according to manufacturer's instructions and verified by PCR and sequencing and titrated using Adeno-X Rapid Titer kits (BD Bioscience).

Transduction of BM-derived DCs with adenoviral vectors. Recombinant adenoviral vectors (ad-shRNA-SOCS1 and ad-shRNA-mSOCS1) were produced and titrated as described above. Mouse bone marrow (BM)-derived DCs (MBDDs) were prepared by Shen et al method (16). In brief, mouse BM was flushed from the hind limbs, passed through a nylon mesh and depleted of red cells using ammonium chloride. After extensive washing with RPMI-1640, the cells were cultured with RPMI-1640 supplemented with 10\% FBS, recombinant mouse granulocyte-macrophage colony-stimulating factor (GM-CSF, $20 \mathrm{ng} / \mathrm{ml}$; PeproTech) and recombinant mouse IL-4 (20 ng/ml; PeproTech). On the 2 nd and 4 th day of culture, the supernatant was removed and replaced with fresh medium containing mGM-CSF and mIL-4. All cultures were incubated at $37^{\circ} \mathrm{C}$ in $5 \%$ humidified $\mathrm{CO}_{2}$. Non-adherent granulocytes were removed after $48 \mathrm{~h}$ and fresh medium was added. After 7 days of culture, flow cytometric analysis showed that $>80 \%$ of the cells expressed characteristic DC-specific markers. The DCs were washed and placed in 12-well plates at a concentration of $10^{5}$ cells/well in $400 \mu \mathrm{l}$ RPMI-1640. The cells were exposed to ad-shRNA-mSOCS1 or ad-shRNA-SOCS1 at 50 MOI. After 8-12-h transduction, the cells were washed with PBS and further incubated in fresh tissue culture medium. The immature BMDCs were then pulsed with the HPVm16E7 protein $(25 \mu \mathrm{g} / \mathrm{ml})$ at $37^{\circ} \mathrm{C}$ for $6 \mathrm{~h}$, followed by stimulation with LPS $(0.5 \mu \mathrm{g} / \mathrm{ml})$ for $24 \mathrm{~h}$ to develop mature BMDCs. The harvested DCs were tested with CD80-flurescein isothiocyanate (FITC), CD-83-FITC and CD86-FITC to confirm DC maturation. 
Flow cytometry acquisition and analysis were performed via a FACS using the Cell Quest software (Becton-Dickinson).

\section{Analysis of SOCS1}

Quantitative real-time PCR analysis of SOCS1. Total RNA was extracted from DCs using the TRIzol reagent (Invitrogen) with $1.0 \mu \mathrm{g}$ for each sample and was reverse-transcribed with random hexamer primers using Super Script First-Strand Synthesis kits (Invitrogen). The expression level of GAPDH was evaluated as an internal control. The target mRNAs were then assessed using the following primers: GAPDH: ACAGTC CATGCCATCACTGCC (sense) and GCCTGCTTCACCACC TTCTTG (anti-sense); SOCS1: TGGTTGTAGCAGCTTGTG TCTGG (sense) and CCTGGTTTGTGCAAAGATACTGGG (anti-sense). Real-time PCR analysis was performed using an ABI 7900HT Sequence Detection System(Applied Biosystems, Foster City, CA, USA) in $20 \mu$ l quadruplicate reactions, using the equivalent of $5 \mathrm{ng}$ of the starting RNA material per reaction as the template.

Western blot analysis of SOCS1 silencing. Since SOCS1 expression in DCs was difficult for western blot analysis, the inhibitory effect of ad-shRNA-SOCS1 and ad-shRNAmSOCS1 were conducted in B16 cells (ATCC, Manassas, VA, USA). The cells were cultured in DMEM and infected by the recombinant adenovirus plasmids for $48 \mathrm{~h}$ at 50 and $100 \mathrm{MOI}$ and then lysed with cell lysis buffer [0.3\% NP40, $1 \mathrm{mM}$ EDTA, $50 \mathrm{mM}$ Tris-Cl (pH 7.4), 2 mM EGTA, $1 \%$ Triton X-100, $150 \mathrm{mM} \mathrm{NaCl}, 25 \mathrm{mM} \mathrm{NaF}, 1 \mathrm{mM} \mathrm{Na} \mathrm{VO}_{3}$, $10 \mu \mathrm{g} / \mathrm{ml}$ phenylmethylsulfonyl fluoride] for $30 \mathrm{~min}$ on ice and then centrifuged at $12,000 \mathrm{x}$ g for $15 \mathrm{~min}$ at $4^{\circ} \mathrm{C}$. The protein samples were separated via SDS-PAGE and transferred onto immobilon membranes (Millipore, MA, USA). SOCS1 and $\beta$-actin proteins were identified using anti-SOCS1 polyclonal and anti- $\beta$-actin monoclonal antibodies (Santa Cruz Biotechnology, Santa Cruz, CA, USA), respectively.

Cytokine ELISA analysis. Levels of various cytokines (IL-12p70, IL-6, TNF- $\alpha$ ) induced by HPV16mE7-pulsed DCs in response to LPS stimulation $(100 \mathrm{ng} / \mathrm{ml})$ for $24 \mathrm{~h}$ were quantitated using the supernatant of DC cultures at the time-points and with the stimulus by ELISA analysis (Dakewe Biotech Co. Ltd., China) according to the manufacturer's instructions. Data are representative of three independent assays.

Tumor models. Female C57BL/6 (H-2 $\left.{ }^{b}\right)$ mice were purchased from Guangzhou Traditional Chinese Medicine University (Guangzhou, China). The mice were housed in filter top cages under specific pathogen-free conditions and used in accordance with the guidelines of Tsinghua University Council on Animal Care. C57BL/6 mice (10 per group) in therapy experiments received a subcutaneous (s.c.) tumor injection of $5 \times 10^{6}$ cells $/ \mathrm{ml}$ of TC-1 cells (ATCC) constitutively expressing wild-type HPV16E6E7 in $100 \mu$ l Hank's buffered salt solution (HBSS, Sigma-Aldrich) for nine days prior to immunization. At day 9, all mice had palpable tumors and then were treated with PBS and various forms of DCs vaccines.

DCs immunization for tumor therapy. Immature BMDCs infected with ad-shRNA-mSOCS1 or ad-shRNA-SOCS1 at
50 MOI were pulsed with the HPVm16E7 protein $(25 \mu \mathrm{g} / \mathrm{ml})$ at $37^{\circ} \mathrm{C}$ for $6 \mathrm{~h}$, followed by stimulation with LPS $(0.5 \mu \mathrm{g} / \mathrm{ml})$ for $6 \mathrm{~h}$ to develop mature BMDCs. The cells $\left(2 \times 10^{5}\right)$ were then washed with PBS to remove extracellular LPS and injected into the hind footpads of C57BL/6 mice inoculated with TC-1 cells expressing HPV16E6E7. The immunized mice were intraperitoneally treated with LPS three times on days 1, 3 and 5 after DC vaccine immunization. The tumor volume was calculated using the following formula: (major axis $\mathrm{x}$ minor axis $\left.^{2}\right) \times 0.52$. The tumor-bearing mice were euthanized when the tumor volume reached $\sim 2,500 \mathrm{~mm}^{3}$.

ELISA for HPV16mE7 antibodies. Enzyme-linked immunosorbent assays (ELISA) were performed by immobilizing $0.5 \mu \mathrm{g}$ HPV16mE7 protein in $50 \mathrm{mM} \mathrm{NaHCO}_{3}(\mathrm{pH} 9.6)$ per microtiter well plate overnight at $4^{\circ} \mathrm{C}$. Blocking was done by $1 \%$ phosphate-buffered saline (PBS) $(\mathrm{pH} 7.5)$ containing $1 \%$ gelatin for $2 \mathrm{~h}$ at $37^{\circ} \mathrm{C}$. Each protein ligand was incubated in PBS ( $\mathrm{pH} \mathrm{7.5)} \mathrm{with} 0.2 \%$ gelatin within the appropriate wells for $1 \mathrm{~h}$ at room temperature at a concentration of $5 \mu \mathrm{g} /$ $\mathrm{ml}$, then anti-HPV16 E7 monoclonal antibody (Santa Cruz Biotechnology) or sera from the immunized mice were added. In the control experiments, the first antigen was omitted from the immobilization step. Incubation with each antibody was carried out in the same solution overnight at $4^{\circ} \mathrm{C}$.

CTL assay. C57BL/6 mice (four per group) were immunized twice in one-week interval with subcutaneous injections of different DCs vaccines at the hind footpads. Control mice received a sham injection of Dulbecco's phosphate-buffered saline (DPBS). Two weeks after DCs inoculation, the mice were euthanized via cervical dislocation. Single-cell suspensions of pooled spleens from each group were prepared in a CTL medium composed of RPMI-1640 (Gibco/BRL, Carlsbad, CA, USA) supplemented with $10 \%$ FCS, 2 mM L-glutamine, $1 \mathrm{mM}$ sodium pyruvate (Gibco/BRL), $50 \mu \mathrm{M}$ 2-mercaptoethanol (Sigma-Aldrich,) and $50 \mu \mathrm{g} / \mathrm{ml}$ gentamicin sulfate (Gibco/BRL). The splenocytes were restimulated in $10 \mathrm{ml} \mathrm{CTL}$ medium by incubating $3 \times 10^{7}$ viable lymphoid cells in $1 \mu \mathrm{M}$ E7.49-53 peptide at an upright T25 tissue culture flask. The effector cells were harvested after a 7-day incubation and then analyzed for cytolytic activity and intracellular $\gamma$ interferon $(\mathrm{IFN}-\gamma)$ production.

$L D H$ release assay. The cytolytic activity was assayed in triplicate in 96-well culture plates by culturing the effector cells with target TC-1 cells expressing HPV16 E6 and E7 proteins at effector/target $(\mathrm{E} / \mathrm{T})$ ratios of $100: 1,33: 1$ or 11:1. After a 4-h incubation at $37^{\circ} \mathrm{C} / 5 \% \mathrm{CO}_{2}$, the 96 -well culture plates were centrifuged at $200 \mathrm{x}$ g for 5 min using a Beckman G5-6R centrifuge (Beckman Coulter Canada, Mississauga, ON, Canada). The culture supernatant $(100 \mu \mathrm{l})$ was collected from each well and transferred into Beckman ready caps and the released LDH activity was determined via biochemical analysis using the Beckman Biochemical analyzer. The target cells cultured without effector cells in either the medium or Triton X-100 (1\% wt/vol) were used to determine the spontaneous or total release of the label. The results were expressed as percent specific lysis, calculated by $\left[\left(\mathrm{LDH}_{\text {test }}-\mathrm{LDH}_{\text {spont }}\right) /\right.$ $\left.\left(\mathrm{LDH}_{\text {total }}-\mathrm{LDH}_{\text {spont }}\right)\right] \mathrm{x}$ 100. The control target $\mathrm{LDH}$ values 
A

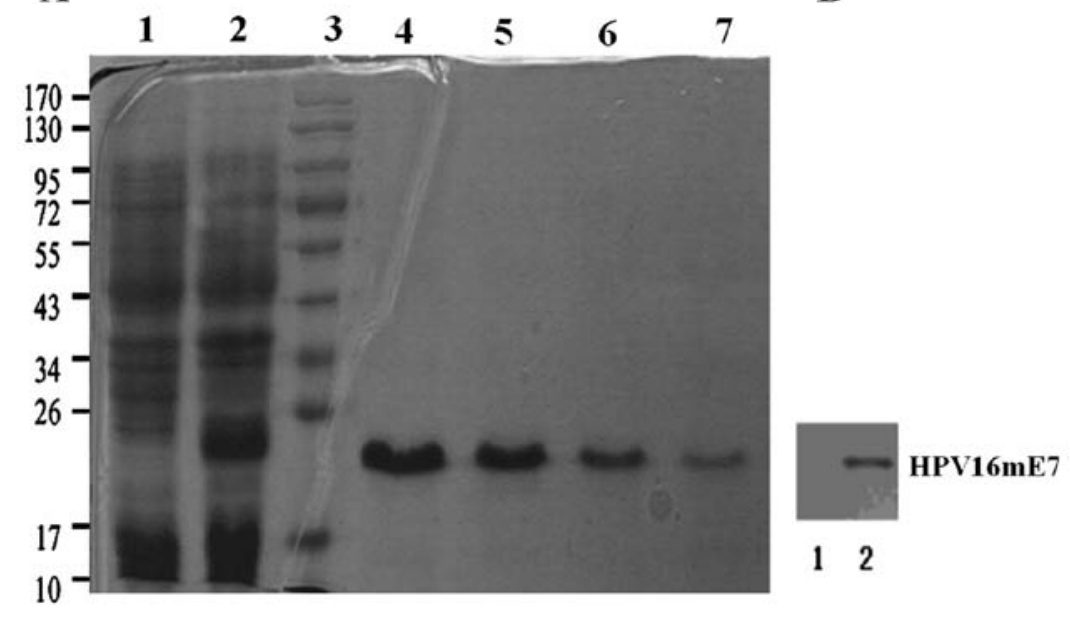

Figure 1. (A) Expression, purification and characterization of HPV16mE7. Lane 1, without IPTG induction; lane 2, with IPTG induction; lane 3, marker; lanes 4-7, 60, 100, 150 and 200 mmol/l imidazole elution buffer, respectively. (B) Western blot analysis for HPV16mE7 using anti-HPV16 E7 monoclonal antibodies. Lane 1, control; lane 2, HPV16mE7.

were consistently $<10 \%$ and were subtracted from the obtained results.

IFN- $\gamma$ ELISA and ELISPOT assay. The intracellular $\gamma$ interferon (IFN- $\gamma$ ) production of the activated antigen-specific cytotoxic $\mathrm{T}$ cells in splenocytes harvested from immunized C57BL/6 mice were detected using the ELISA kit and ELISPOT kit (eBioscience Co.).

IFN- $\gamma$ ELISA assay. C57BL/6 mice (four per group) were immunized twice with one week interval with PBS, DCs, DC-ad-shRNA-SOCS1, or DC-ad-shRNA-mSOCS1. The DCs were HPV16mE7 pulsed. After 7 days post-immunization, pooled splenocytes from each group were re-stimulated with E7.49-57 for 7 days. The effector cells was cultured with target TC-1 cells which express HPV16 E6 and E7 proteins at effector/target $(\mathrm{E} / \mathrm{T})$ ratios of 100:1, 33:1 or 11:1. The IFN- $\gamma$ ELISA assay was performed following the instruction manual.

IFN- $\gamma$ ELISPOT assay. In brief, on day 9 of post-immunization, the 96 -well nitrocellulose-base plates were coated overnight at $4{ }^{\circ} \mathrm{C}$ with purified anti-mouse IFN- $\gamma$ antibodies and then blocked with the complete media. Splenocytes $(100 \mu \mathrm{l} /$ well) cultured in RPMI-1640 (Gibco/BRL) were seeded in the wells at an initial concentration of $10^{6}$ cells/well and a row of serial dilutions either unstimulated or stimulated with E7.49-57 or R187 peptide $(1 \mu \mathrm{g} / \mathrm{ml})$ or buffer were prepared. PMA (5 ng/ml, Sigma) served as a positive control and the R187 peptide or media served as a negative control. The plate was incubated overnight at $37^{\circ} \mathrm{C} / 5 \% \mathrm{CO}_{2}$ and then detected by detection antibody (a biotinylated anti-mouse IFN- $\gamma$ antibody) for $2 \mathrm{~h}$ at room temperature. After removing the unbound detection antibody, the enzyme conjugate (streptavidin-HRP) was added. The unbound streptavidin-HRP was washed off after a 1-h incubation at room temperature and the plate was stained with an AEC substrate solution for 20 min. Further, the plate was washed and air-dried overnight. Foci of staining were counted using a magnifying lens.

Statistical analysis. All data were expressed as means \pm standard deviation (SD) and were representative of at least two different experiments. The statistical significance of group differences was measured by Student's t-test. p-values $<0.05$ were considered to be significant. The mouse survival rates were analyzed using the Kaplan-Meier method (log-rank test).

\section{Results}

Construction and characterization of recombinant HPV16mE7. HPV16mE7 was cloned into pET-28a vector, expressed in BL21 $\gamma$ (DE3) E. coli and purified to homogeneity to allow in vitro/in vivo assays (Fig. 1A). An LPS elimination procedure was introduced in the purification protocol. A series of diluted imidazole elution buffer, 60, 100, 150 and $200 \mathrm{mM}$, respectively, were used to determine the best elution concentration. The presence of the E7 protein in HPV16mE7 was confirmed via western blot analysis using an anti-HPV16 E7 monoclonal antibody or anti-His monoclonal antibody (Fig. 1B). HPV16 E7 concentration was measured by BCA kit (Beyotime, China) according to the instruction manual for the following assays.

Inhibition of SOCS1 expression. Constructs of ad-shRNASOCS1 and ad-shRNA-mSOCS1 were verified in B16 cells (Fig. 2A). Western blot analysis showed that ad-shRNASOCS1 (MOI, 50 or 100) infection greatly reduced SOCS1 expression compared with infection by ad-shRNA-mSOCS1 (MOI, 50). Real-time RT-PCR analysis also confirmed the inhibition of SOCS1 expression in DCs (Fig. 2B). The SOCS1 mRNA level was reduced significantly by ad-shRNA-SOCS1 but not by ad-shRNA-mSOCS1. Ad-shRNA-SOCS1 inhibited the SOCS1 mRNA level, however, ad-shRNA-mSOCS1 failed. Furthermore, the inhibition by ad-shRNA-SOCS1 at MOI 100 was better than that at MOI 50 .

Analysis of DC maturation. Immature DCs (iDCs) were produced from mouse bone marrow via stimulation with GM-CSF and IL-4 for 7 days and further stimulation with ad-shRNA-SOCS1. The expression of the surface markers CD80, CD83 and CD86 were analyzed using flow cytometry 
A

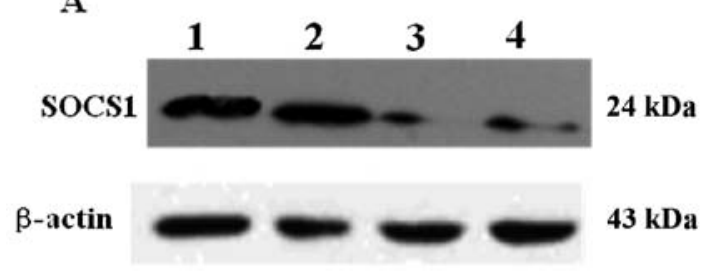

B

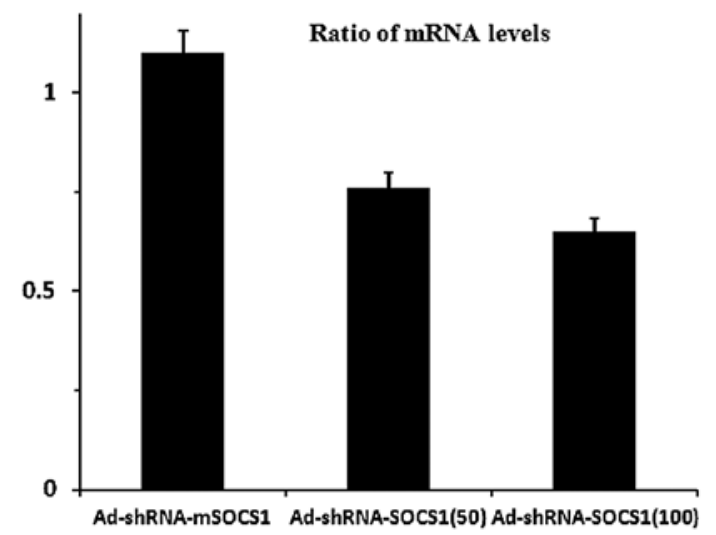

Figure 2. (A) Western blot analysis showing the inhibition of SOCS1 expression in B16 cells. Lane 1, PBS; lane 2, Ad-shRNA-mSOCS1 (MOI 50); lane 3, Ad-shRNA-SOCS1 (MOI 100); lane 4, Ad-shRNA-SOCS1 (MOI 50). (B) The SOCS1 mRNA levels were normalized to that of GADPH in DCs (values are mean SEM). DCs were infected by ad-shRNA-mSOCS1 (MOI 50), ad-shRNA-SOCS1 (MOI 50) and ad-shRNA-SOCS1 (MOI 100).

before and after $24 \mathrm{~h}$ LPS stimulation and analyzed by SPSS one-way ANOVA (Table I). Results in Table I showed that the expression levels of all three antigens significantly increased by 2-4-fold during maturation in both ad-shRNA-SOCS1 and ad-shRNA-mSOCS1 infected DCs with respect to control. The former increased $10 \%$ more than the latter. In addition, although LPS greatly induced the expression of these CD markers in BMDC, it was lower than that of the ad-shRNASOCS1, and the ad-shRNA-mSOCS1 group.

Cytokine ELISA analysis in DCs. Levels of various proinflammatory cytokines such as IL-12, IL-6 and tumor necrosis factor (TNF)- $\alpha$ (IL-12p70, IL-6, TNF- $\alpha$ ) induced by HPV16mE7-pulsed DCs with or without LPS stimulation were
Table I. Percentage of DCs expressing CD80, CD83 and CD86.

\begin{tabular}{lccl}
\hline & CD80 & CD83 & CD86 \\
\hline Control & $27.27 \pm 6.3$ & $38.8 \pm 6.5$ & $26.5 \pm 5.5$ \\
LPS & $72.5 \pm 8.3^{\mathrm{a}}$ & $78 \pm 9.2^{\mathrm{a}}$ & $73.3 \pm 7.2^{\mathrm{a}}$ \\
Ad-shRNA-mSOCS1 & $75.2 \pm 8.7^{\mathrm{a}, \mathrm{b}}$ & $80.3 \pm 6.2^{\mathrm{a}, \mathrm{b}}$ & $78.3 \pm 4.5^{\mathrm{a}, \mathrm{b}}$ \\
Ad-shRNA-SOCS1 & $88.6 \pm 7.1^{\mathrm{a}, \mathrm{c}}$ & $92.3 \pm 4.8^{\mathrm{a}, \mathrm{c}}$ & $95.5 \pm 2.8^{\mathrm{a}, \mathrm{d}}$ \\
\hline
\end{tabular}

Immature DCs were stimulated by GM-CSF and IL-4 for 7 days and further stimulated by ad-shRNA-SOCS1 or ad-shRNA-mSOCS1. Percent of DCs expressing CD80, CD83 and CD86 were detected using flow cytometry before and after $24 \mathrm{~h}$ LPS stimulation and analyzed by SPSS one-way ANOVA. a Significant differences compared with that of the DC control, $\mathrm{p}<0.001$; ${ }^{\mathrm{b}}$ no significant differences compared with that of the LPS control, $\mathrm{p}>0.05$; ' ${ }^{\mathrm{s}}$ ignificant differences compared with that of the LPS control or Ad-shRNA-mSOCS1 group, $\mathrm{p}<0.05$; ${ }^{\mathrm{d}}$ significant differences compared with that of the LPS control, $\mathrm{p}<0.05$, but no significant differences compared with that of the Ad-shRNA-mSOCS1 group, $\mathrm{p}>0.05$.

quantitated by ELISA analysis kits (Fig. 3). Silencing SOCS1 in HPV16mE7-pulsed DCs drastically enhanced the production of cytokines, such as IL-12p70, IL-6 and TNF- $\alpha$ with LPS stimulation, but not in the absence of LPS stimulation. By contrast, the PBS control group and the ad-shRNA-mSOCS1 group expressed far less cytokines with or without LPS stimulation.

Immune antibodies induced by different $D C$ vaccines. Four mice from each group were immunized using $10^{5} \mathrm{DC}$-treated vaccine or $5 \mu \mathrm{g}$ HPV16 E7 in order to determine the HPV16 E7 antibody level induced by different DC vaccines. After two weeks, the HPV16 E7 antibody level in the serum was measured by ELISA. Antibody responses which were induced by different vaccines were compared with PBS controls. DCs-ad-shRNA-SOCS1 induced the strongest response, with antibody titers 5- and 1.5-fold higher than those of the HPV16mE7 vaccine and DC-ad-sh-mSOCS1, respectively (Fig. 4). Our results suggest that SOCS1-silenced HPV16mE7-pulsed DCs promoted the production of specific antibodies.
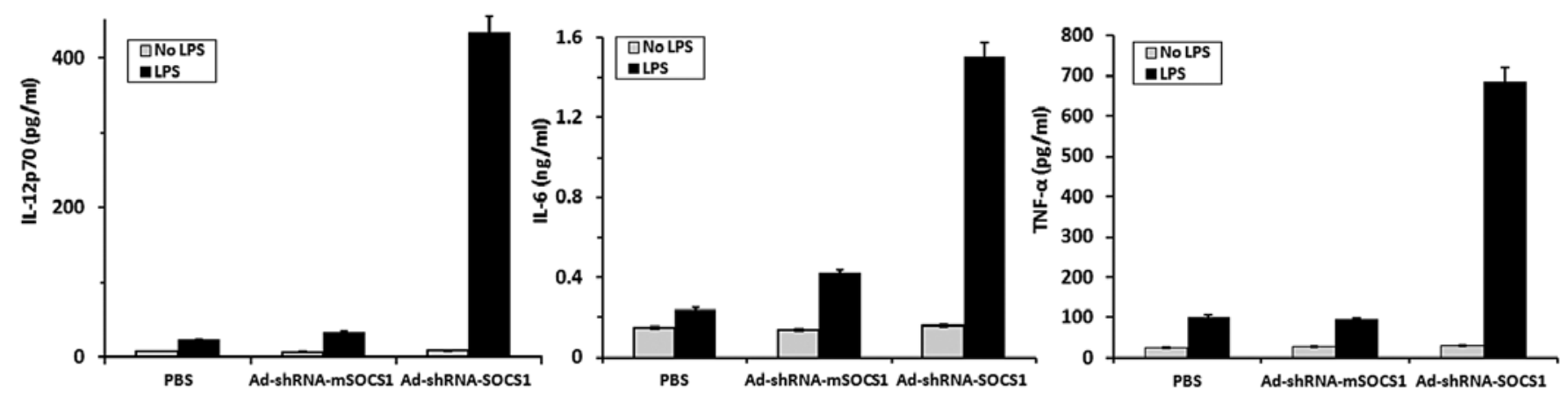

Figure 3. SOCS1 negatively regulates BM-derived DCs in response to LPS stimulation. Cytokines were secreted by transfected DCs after LPS (100 ng/ml) stimulation. Stimulation for $24 \mathrm{~h}$ was measured via ELISA assays. Data are representative of three independent assays. "p<0.01 versus SOCS1-siRNA DCs. 


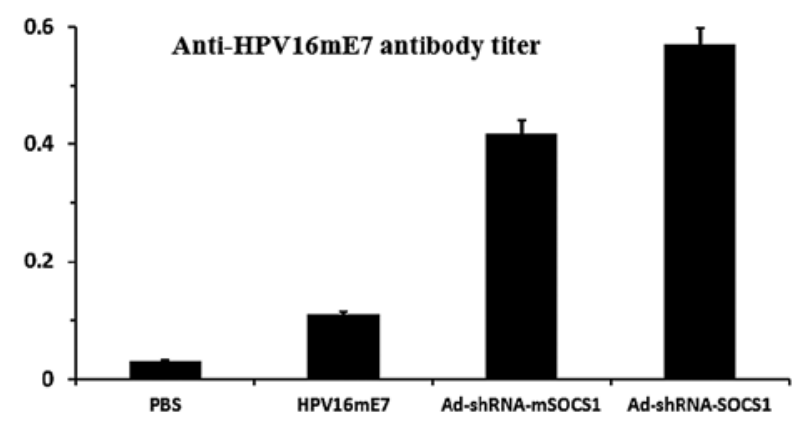

Figure 4. Anti-HPV16 E7 antibody levels of the different vaccinations. Four mice from each group were treated with PBS, HPV16mE7, DCs-ad-shRNAmSOCS1, DCs-ad-shRNA-SOCS1 to determine the HPV16 E7 antibody level.

$L D H$ release assay. C57BL/6 mice (four per group) were immunized at the footpads with DCs, ad-shRNA-mSOCS1 pulsed with E7.49-57, ad-shRNA-SOCS1 pulsed with E7.49-57 and ad-shRNA-SOCS1 pulsed with HPVmE7, respectively, to determine whether DC vaccines could induce CTL responses against HPV16 E7. After 7 days of post-immunization, pooled splenocytes from each group were restimulated with $1 \mu \mathrm{g} / \mathrm{ml}$ E7.49-57 for 7 days. Their specific lytic activities against TC-1 cells at 100:1, 33:1 and 11:1 E/T ratios were assayed using an $\mathrm{LDH}$ release assay (Fig. 5). Immunization of C57BL/6 mice with DC-ad-shRNA-SOCS1 (HPVmE7) induced the strongest specific CTL responses to TC-1 cells than that pulsed with E7.49-57 and controls (DCs and ad-shRNA-mSOCS1 pulsed with E7.49-57), respectively. The lysis effect induced by ad-shRNA-SOCS1 (E7.49-57) and ad-shRNA-SOCS1 (HPVmE7) were not linear, but it increased with effector:target ratio compared with the linear increase of the controls.

ELISA analysis and ELISPOT of IFN- $\gamma$ expression. ELISA analysis was used for the detection of IFN- $\gamma$ in the supernatant of the same restimulated splenocytes. C57BL/6 mice (four per group) were immunized twice in 1-week interval with PBS, DCs, DC-ad-shRNA-SOCS1, or DC-ad-shRNA-mSOCS1, respectively. The DCs were all pulsed by HPV16mE7. Seven days post-immunization, pooled splenocytes from each group were re-stimulated with E7.49-57 for 7 days. The released IFN- $\gamma$ in the supernatant at 100:1, 33:1 and 11:1 E/T ratio was assayed (Fig. 6A). Results showed that the expression level of IFN- $\gamma$ was greatly enhanced by silencing SOCS1, whereas similar effect was observed in ad-shRNA-mSOCS1 and non-treated DC cells. ELISPOT of IFN- $\gamma$ in response to in vitro stimulation with HPV16mE7 was quantified to estimate the frequencies of HPV16-E7-specific splenocytes in mice immunized with DC vaccines (Fig. 6B). The detection efficiency of the positive control, PMA $(5 \mathrm{ng} / \mathrm{ml})$ was $95 \%$ (data not shown). Our results also showed that ad-shRNA-SOCS1 group had a significant effect in inducing IFN- $\gamma$ expression when stimulated by E7.49-57 peptide. Further, Ad-shRNAmSOCS1 group expressed high level of IFN- $\gamma$, compared with the buffer group, and the R187 group.

Efficacy of the DC vaccine in treating mice bearing tumors. The ability of the DC-ad-shRNA-SOCS1 vaccine to treat mice bearing tumors was investigated in the groups of $10 \mathrm{C} 57 \mathrm{BL} / 6$

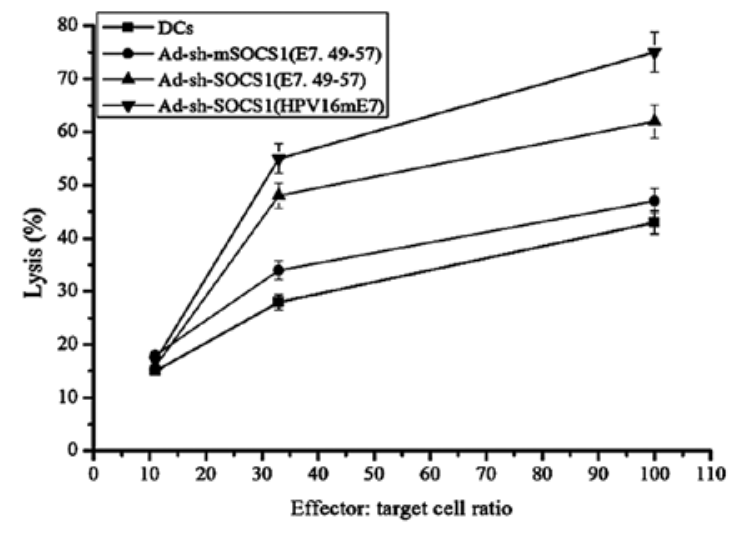

Figure 5. LDH release assay. C57BL/6 mice were immunized via injection into the hind footpads of C57BL/6 mice with DCs, DC-ad-shRNA-mSOCS1 (E7.49-57), DC-ad-shRNA-SOCS1 (E7.49-57) and DC-ad-shRNA-SOCS1 (HPV16mE7). The peptides pulsed in the DCs were given in the parentheses. At seven days post-immunization, pooled splenocytes from each group were restimulated with E7.49-57 for seven days. Their specific lytic activities against TC-1 cells at 100:1, 33:1 and 11:1 E/T ratios were assayed.
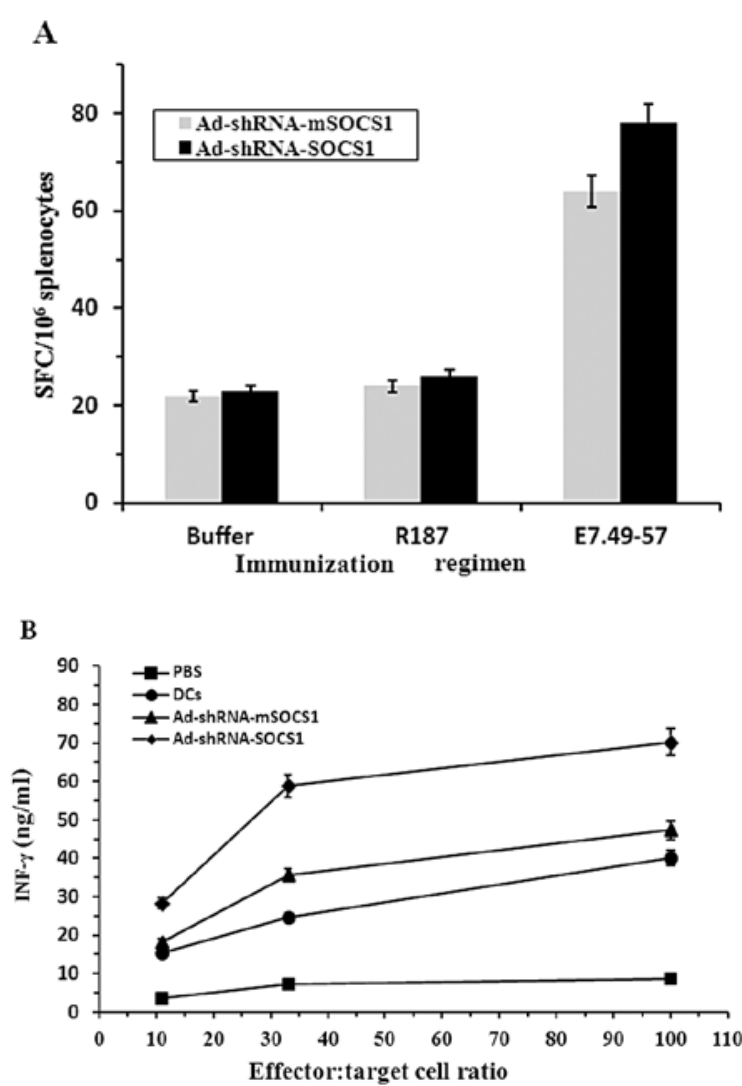

Figure 6. (A) ELISPOT IFN- $\gamma$. The IFN- $\gamma$-producing cells were assayed ex vivo via IFN- $\gamma$ ELISPOT using freshly isolated splenocytes seven days after the single or boost immunization. Data shown are mean standard deviations for two independent assays. (B) IFN- $\gamma$ ELISA. C57BL/6 mice were immunized twice with PBS, DCs, ad-shRNA-SOCS1, or ad-shRNAmSOCS1. At seven days post-immunization, pooled splenocytes from each group were restimulated with E7.49-57 for seven days. The released IFN- $\gamma$ in the culture supernatant at 100:1, 33:1 and 11:1 E/T ratio was assayed.

mice which received $5 \times 10^{5} \mathrm{TC}-1$ tumor cells. At day 9 , all mice had palpable tumors and therapeutic treatments were initiated with DC-ad-shRNA-SOCS1, DC-ad-shRNA-mSOCS1, DCs 
A

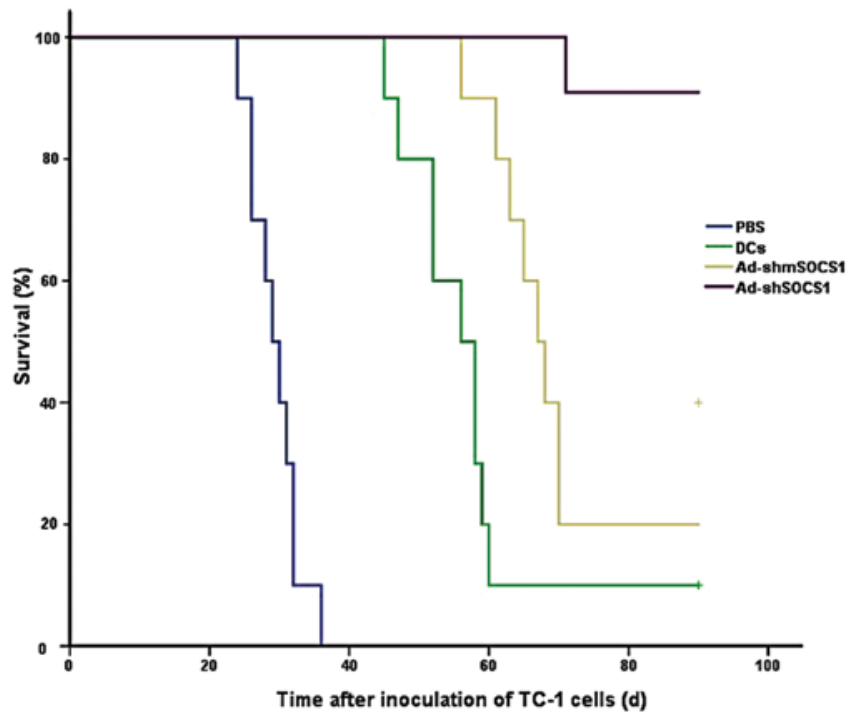

B

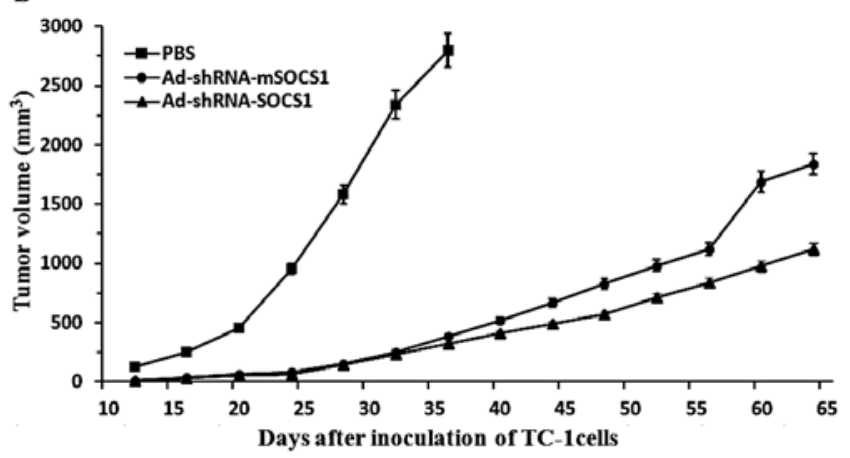

Figure 7. (A) Survival rate of mice with established tumors. Groups of $10 \mathrm{C} 57 \mathrm{BL} / 6$ mice were injected with $5 \times 10^{5} \mathrm{TC}-1$ tumor cells and the therapy was initiated with PBS, DCs, DCs-ad-shRNA-mSOCS1 or DCsad-shRNA-SOCS1 on day 9. Data of the survival rates after inoculation of TC-1 cells were analyzed using the Kaplan-Meier method (log-rank test). " $\mathrm{p}<0.01$ (Ad-shRNA-SOCS1 vs PBS or Dcs or Ad-shRNA-mSOCS1), $\mathrm{p}<0.01$ (Ad-shRNA-mSOCS1 or DCs vs PBS). (B) The curve of tumor volume and time. Groups of 10 C57BL/6 mice were injected with $5 \times 10^{5} \mathrm{TC}-1$ tumor cells and the therapy was initiated with PBS, DCs, DCs-ad-shRNA-mSOCS1 or DCs-ad-shRNA-SOCS1 on day 9 after tumor injection. Data of the tumor volume after inoculation of TC-1 cells were analyzed using Student's t-test. " $\mathrm{p}<0.01$ (Ad-shRNA-SOCS1 or Ad-shRNA-mSOCS1 or DCs vs PBS) $\mathrm{p}<0.05$ (Ad-shRNA-SOCS1 vs Ad-shRNA-mSOCS1 or DCs).

and PBS, respectively. The vaccination with DC-ad-shRNASOCS1 (Fig. 7A) showed $100 \%$ survival rate until day 68 , while all mice died on days 36, 62 and 72 in the PBS, DCs and DC-ad-shRNA-mSOCS1 group, respectively. Tumor volume and time experiment showed that DCs-ad-shRNASOCS1 vaccination significantly decreased the tumor volume compared with vaccination with controls (Fig. 7B). The experiments were repeated, producing a composite $\mathrm{p}$-value $(\mathrm{p}<0.01)$. Although therapeutic vaccination could not always eradicate the tumor completely, the survival rate was significantly enhanced in mice for $>80$ days (Fig. 7).

\section{Discussion}

In the present study, the HPV16mE7 protein-pulsed DC vaccine was based on the E7 oncogene of HPV16, which is thought as the gold standard for cervical cancer immune therapy, with some therapeutic vaccines under development in preclinical models (27). Experiments on the DNA vaccination with E7 oncogene, however, still harbor the risk of transformation for the cells that receive and express the oncogene $(28,29)$. In this study, HPV-16E7 gene was modified to resolve the interference with binding to the host cell $\mathrm{Rb}$ protein and by E7 gene mutation to reduce the transformation capacity (22). Western blot analysis (Fig. 1B) and anti-HPV16 E7 antibody titer test (Fig. 4) demonstrated that the mutated HPV16 E7 possessed antigenicity of wild-type HPV16 E7. Our results (Figs. 3 and 7) confirmed that the HPV16mE7 based vaccine strategies dramatically enhanced the expression levels of various cytokines, retarded the tumor growth and prolonged the survival time of the mouse models in therapeutic experiments and improved CTL mediated lysis compared with the controls. In addition, vaccination with the modified HPV16 E7 showed obvious advantages in inducing immune responses compared to vaccination with the wild-type as described in a previous study (23). Thus, the modified HPV16 E7 proteinpulsed DC vaccine could be a safe and effective vaccine in treating HPV-associated diseases.

The antigen-presenting DCs were able to induce robust cell-mediated immunity capable of attacking and eliminating abnormal antigen-bearing cells. DC vaccine pulsed with the HPV16 E7 oncoprotein exhibits significant advantages of potentially presenting multiple immunogenic CTL and antibody responses (30-33). Clinical studies in patients with human papillomavirus cervical cancers demonstrated by vaccinating with HPV E7-pulsed dendritic cells that HPVE7loaded DC vaccination is safe and immunogenic for stage IB or IIA cervical cancer patients (32). DC vaccination was well tolerated and no significant toxicities were recorded. Similar results were obtained by Ferrara et al (33). How to fully activate DCs as a vaccine is the key to cancer immunotherapy. One strategy to achieve immunotherapy is to inhibit the negative regulatory pathways during DC activation which enhances cancer immunity and breaks self-tolerance (8). In this study, we knocked down SOCS1, a negative signaling regulator of various cytokines in DCs. The efficiency of SOCS1 silencing was verified in B16 and DCs (Fig. 2) and the inhibition by ad-shRNA-SOCS1 at MOI 100 was better than that at MOI 50, indicating a dosage-dependent manner. Silencing SOCS1 also enhanced the expression levels of IL-12p, IL-6, TNF- $\alpha$ and IFN- $\gamma$ (Figs. 3 and 6). Our finding is consistent with previous reports that SOCS1 represents an inhibitory mechanism for qualitatively and quantitatively controlling antigen presentation by DCs and the magnitude of adaptive immunity $(11,12,16-18)$. Hence, this study underscores the critical role of SOCS1 silencing in the stimulation of DC immune response and indicates that SOCS1 silencing can be applied for a large number of diseases in addition to HPV diseases.

An important finding of this study is that DCs-ad-shRNASOCS1 induced specific anti-HPV16mE7 antibody and CTL responses. Although the mechanism on how the SOCS1 silencing induced the priming of antigen-specific CTLs is not clear, SOCS1-restricted DCs greatly enhanced the secretion of pro-inflammatory cytokines, such as IL-12, IL-6 and TNF- $\alpha$ (Fig. 3) and promoted the production of specific antibodies (Fig. 4). Animal experiments demonstrated that the vaccine 
with SOCS1 silencing possessed a high ability to induce CTLs (Fig. 5) and enhanced IFN- $\gamma$ expression (Fig. 6B). These data are consistent with observations of Hashimoto et al in mouse DCs (34) and the findings of the CTL response generated against HIV (12). Song et al found that SOCS1 silencing in DCs enhanced production of a mixed pattern of Th1-and Th2-polarizing cytokines (12). Further, Kelvin et al pointed out that persistent antigen presentation of DCs to induce pathological autoimmune responses against normal tissues and tumor could be achieved by silencing SOCS1 to unleash the signaling of IL-12 (11). Previous studies by Hong et al showed that the addition of anti-IL-6 antibody into the DC:T-cell co-culture also blocked the immuno-stimulatory function of siSOCS1 DCs, albeit with a lower efficiency (17). Interestingly, the study also found that the control vector Ad-shRNA-mSOCS1 showed high levels of DC markers, anti-HPV antibody and IFN- $\gamma$-producing splenocytes compared with DCs-ad-shRNASOCS1 (Table I, Figs. 4 and 6A), indicating a non-specific stimulatory effect of siRNA molecules via the activation of TLR signaling and some cellular genes (35-37) or possibly due to the toxicity of the replication-incomplete adenovirus vector-specific response $(38,39)$, or both. However, the level of non-specific immune caused by SOCS1 silencing was low compared with that by CTLA-4 (another immunosuppressive) (16) and could achieve a more antigen-specific antitumor response. Results in Fig. 6 indicate that SOCS1 silencing had no obvious function in inducing IFN- $\gamma$-producing splenocytes, but significantly enhanced IFN- $\gamma$ expression in these cells. The possible explanation for the marginal difference of IFN- $\gamma$-producing cells between DCs-ad-shRNA-SOCS1 and DCs-ad-shRNA-mSOCS1 in Fig. 6A was that silencing SOCS1 has limited functions in promoting antigen presentation and splenocyte activation, which was in accordance with the results in Table I. However, there is no direct evidence that SOCS1 is involved in antigen presentation and splenocytes activation or what part it takes. More research is needed for further explanation. In addition, our study confirmed that DCs with SOCS1 silencing were hyper-responsive to lipopolysaccharide (LPS) (Fig. 3), which was thought to interact with Toll-like receptor (TLR) 4 for signaling and involved in the stimulation of DC maturation and proinflammatory cytokine expression. Thus, the immune responses induced by DC-adshRNA-SOCS1 may be a collective result of specific and non-specific immunity as well as the enhanced sensitivity to LPS as discussed by Song et al (12).

This study, to our knowledge, first underscores the immunotherapeutic effect by combining the HPV16mE7 protein and silencing SOCS1 in DCs as a vaccine in treating HPV. The vaccine showed significant immune effect by inducing complex immune responses against HPV and greatly prolonged the lifetime of the mouse models. Thus, the strategy of silencing SOCS1 in HPV16mE7 protein-pulsed DCs may open a new and alternative avenue to develop safe and effective therapeutic HPV and other infectious disease vaccines.

\section{Acknowledgements}

We would like to thank Dr Xiaosong Song and other members of Huang Lab for suggestions on the study and assistance in revising the manuscript. This work was supported in part by funding from Shenzhen Municipal Government and Bureau of Science, Technology and Information (grants 2006464, 200712, SG200810150043A, CXB201005260070A, and CXB201104220043A to L.H.; and JC200903180532A to Y.Z.), RFDP (20090002120055 to Y.Z.), and Nanshan District Bureau of Science and Technology.

\section{References}

1. Jemal A, Bray F and Center MM: Global cancer statistics. CA Cancer J Clin 61: 69-90, 2011.

2. Berzofsky JA, Terabe M, Oh SK, Belyakov IM, Ahlers JD, Janik JE and Morris JC: Progress on new vaccine strategies for the immunotherapy and prevention of cancer. J Clin Invest 113: $1515-1525,2004$.

3. Ali M and Monk BJ: Vaccines against human papillomavirus and cervical cancer: promises and challenges. Oncologist 10: 528-538, 2005.

4. Kanodia S, Da Silva DM and Kast WM: Recent advances in strategies for immunotherapy of human papillomavirus-induced lesions. Int J Cancer 122: 247-259, 2008.

5. Psyrri A and Daniel D: Human papillomavirus in cervical and head-and-neck cancer. Nat Clin Pract Oncol 5: 24-31, 2008.

6. Brinton LA: Epidemiology of cervical cancer: overview. IARC Sci Publ 119: 3-23, 1992.

7. Zur Hausen H: Papillomaviruses and cancer: from basic studies to clinical application. Nat Rev Cancer 2: 342-350, 2002.

8. Kobayashi T and Yoshimura A: Keeping DCs awake by putting SOCS1 to sleep. Trends Immunol 26: 177-179, 2005.

9. Santin AD, Hermonat PL, Ravaggi A, Chiriva-Internati M, Zhan D and Pecorelli S: Induction of human papillomavirusspecific CD4(+) and CD8(+) lymphocytes by E7-pulsed autologous dendritic cells in patients with human papillomavirus type 16- and 18-positive cervical cancer. J Virol 73: 5402-5410, 1999.

10. Kim TW, Lee JH, He L, Boyd DA, Hardwick JM and Hung CF: Modification of professional antigen-presenting cells with small interfering RNA in vivo to enhance cancer vaccine potency. Cancer Res 65: 309-316, 2005.

11. Kelvin EK, Song XT, Aldrich M, Huang XF and Chen SY: SOCS1 restricts dendritic cells' ability to break self tolerance and induce antitumor immunity by regulating IL-12 production and signaling. J Clin Invest 116: 90-100, 2006.

12. Song XT, Evel-Kabler K, Rollins L, Aldrich M, Gao F, Huang XF and Chen SY: An alternative and effective HIV vaccination approach based on inhibition of antigen presentation attenuators in dendritic cells. PLoS Med 3: 76-93, 2006.

13. Akita H, Kogure K, Moriguchi R, Nakamura Y and Higashi T: Nanoparticles for ex vivo siRNA delivery to dendritic cells for cancer vaccines: Programmed endosomal escape and dissociation. J Control Release 143: 311-317, 2010.

14. Kubo M, Hanada T and Yoshimura A: Suppressors of cytokine signaling and immunity. Nat Immunol 4: 1169-1176, 2003.

15. Alexander WS and Hilton DJ: The role of suppressors of cytokine signaling (SOCS) proteins in regulation of the immune response. Annu Rev Immunol 22: 503-529, 2004.

16. Shen L, Evel-Kabler K, Strube R and Chen SY: Silencing of SOCS1 enhances antigen presentation by dendritic cells and antigen-specific anti-tumor immunity. Nat Biotechnol 22: 1546-1553, 2004.

17. Hong B, Ren W, Song XT and Evel-Kabler K: Human suppressor of cytokine signaling 1 controls immunostimulatory activity of monocyte-derived dendritic cells. Cancer Res 69: 8076-8084, 2009.

18. Hanada T, Yoshida H, Kato S, Tanaka K and Masutani K: Suppressor of cytokine signaling-1 is essential for suppressing dendritic cell activation and systemic autoimmunity. Immunity 19: 437-450, 2003

19. Guenterberg KD, Lesinski GB, Mundy-Bosse BL and Karpa VI: Enhanced anti-tumor activity of interferon-alpha in SOCS1deficient mice is mediated by $\mathrm{CD} 4^{+}$and $\mathrm{CD} 8^{+} \mathrm{T}$ cells. Cancer Immunol Immunother 60: 1281-1288, 2011.

20. Carow B, Ye Xq, Gavier-Widén D, Bhuju S and Oehlmann W: Silencing suppressor of cytokine signaling-1 (SOCS1) in macrophages improves Mycobacterium tuberculosis control in an IFN- $\gamma$-dependent. J Biol Chem 286: 26873-26887, 2011. 
21. Münger K, Basile JR, Duensing S, Eichten A and Gonzalez SL: Biological activities and molecular targets of the human papillomavirus E7 oncoprotein. Oncogene 20: 7888-7898, 2001.

22. Cassetti MC, McElhiney SP, Shahabi V, Pullen JK and Le Poole IC: Antitumor efficacy of Venezuelan equine encephalitis virus replicon particles encoding mutated HPV16E6 and E7 genes. Vaccine 22: 520-527, 2004.

23. Brinkman JA, Xu XM and Kast WM: The efficacy a DNA vaccine containing inserted and replicated regions of the E7 gene for treatment of HPV 16 induced tumors. Vaccine 25: 3437-3444, 2007.

24. Feltkamp MC, Smits HL, Vierboom MP, Minnaar RP, de Jongh BM and Drijfhout JW: Vaccination with cytotoxic $\mathrm{T}$ lymphocyte epitope containing peptide protects against a tumor induced by human papillomavirus type 16-transformed cells. Eur J Immunol 23: 2242-2249, 1993.

25. Liu HG, Fan ZP, Chen WW, Yang HY, Liu QF and Zhang H: A mutant HBs antigen (HbsAg) 183-191 epitope elicits specific cytotoxic T lymphocytes in acute hepatitis B patients. Clin Exp Immunol 151: 441-447, 2008.

26. Zheng Y, Zhang Y, Ma Y, Wan J and Shi C: Enhancement of immunotherapeutic effects of HPV16E7 on cervical cancer by fusion with CTLA4 extracellular region. J Microbiol 46: 728-736, 2008.

27. Brinkman JA, Caffrey AS and Muderspach LI: The impact of anti HPV vaccination on cervical cancer incidence and HPV induced cervical lesions: consequences for clinical management. Eur J Gynaecol Oncol 26: 129-142, 2005.

28. Münger K, Phelps WC, Bubb V and Howley PM: The E6 and E7 genes of the human papillomavirus type 16 together are necessary and sufficient for transformation of primary human keratinocytes. J Virol 63: 4417-4421, 1989.

29. Phelps WC, Yee CL and Munger K: The human papillomavirus type 16 E7 gene encodes transactivation and transformation functions similar to those of adenovirus E1A. Cell 53: 539-547, 1988.
30. Nonn M, Schinz M, Zumbach K, Pawlita M and Schneider A Dendritic cell-based tumor vaccine for cervical cancer I: in vitro vaccination with recombinant protein pulsed dendritic cells induces specific T cells to HPV16 E7 and HPV18 E7. J Cancer Res Clin Oncol 129: 511-520, 2003.

31. Santin AD, Bellone S, Gokden M and Cannon MJ: Vaccination with HPV-18 E7-pulsed dendritic cells in a patient with metastatic cervical cancer. N Engl J Med 346: 1752-1753, 2002.

32. Santin AD, Bellone S, Palmieri M, Zanolini A, Ravaggi A and Siegel ER: Human papillomavirus type 16 and 18 E7-pulsed dendritic cell vaccination of stage IB or IIA cervical cancer patients: a phase I escalating-dose trial. J Virol 82: 1968-1979, 2008.

33. Ferrara A, Nonn M, Sehr P, Schreckenberger C and Pawlita M Dendritic cell-based tumor vaccine for cervical cancer II: results of a clinical pilot study in 15 individual patients. J Cancer Res Clin Oncol 129: 521-530, 2003.

34. Hashimoto M, Ayada T, Kinjyo I, Hiwatashi K and Yoshida H: Silencing of SOCS1 in macrophages suppresses tumor development by enhancing antitumor inflammation. Cancer Sci 100: 730-736, 2009.

35. Judge AD, Sood V, Shaw JR, Fang D and McClintock K: Sequence-dependent stimulation of the mammalian innate immune response by synthetic siRNA. Nat Biotechnol 23: 457-462, 2005.

36. Hornung V, Guenthner-Biller M, Bourquin C and Ablasser A: Sequence-specific potent induction of IFN-alpha by short interfering RNA in plasmacytoid dendritic cells through TLR7. Nat Med 11: 263-270, 2005

37. Sledz CA, Holko M, de Veer MJ and Silverman RH: Activation of the interferon system by short-interfering RNAs. Nat Cell Biol 5: 834-839, 2003.

38. Basak SK, Kiertscher SM and Harui A: Modifying adenoviral vectors for use as gene-based cancer vaccines. Viral Immunol 17: 182-196, 2004.

39. Benihoud K, Yeh P and Perricaudet M: Adenovirusvectors for gene delivery. Biotechnology 10: 440-447, 1999. 